\title{
Equivalencia del Axioma de Elección con el Problema de Redefinición de Funciones
}

\author{
Merino, Andrés $1, *$ iD ; Trujillo, Juan C. ${ }^{2}$ \\ ${ }^{1}$ Escuela de Ciencias Físicas y Matemática. Facultad de Ciencias Exactas y Naturales. Pontificia Universidad Católica \\ del Ecuador. Quito, Ecuador \\ email: aemerinot@puce.edu.ec \\ 2 Departamento de Matemática, Facultad de Ciencias. Escuela Politécnica Nacional. Quito, Ecuador \\ email: juancarlos.trujillodepn.edu.ec
}

\begin{abstract}
Resumen: En el presente trabajo se estudia el problema de redefinir el dominio de una función real dada para que esta sea inyectiva, desde el caso más general, hasta llegar a las restricciones necesarias para que este Problema sea equivalente al Axioma de Elección.
\end{abstract}

Palabras claves: Axioma de Elección, funciones inyectivas, redefinición de funciones.

\section{Equivalence of the Axiom of Choice with the Problem of the Redefinition of Functions}

\begin{abstract}
In this paper we will study the problem of redefining the domain of a given real function in order to obtain an injective function, from the most general case, to the necessary restrictions to make this problem equivalent to the Axiom of Choice.
\end{abstract}

Keywords: Axiom of Choice, injective functions, redefinition of functions.

\section{INTRODUCCIÓN}

En las notas de clase de un curso de Pre-cálculo no publicadas formalmente por alguna editorial o revista científica, pero de amplio uso en cursos de nivelación para el ingreso a las carreras de ingeniería en Ecuador, los autores de este artículo encontramos un ejercicio planteado incorrectamente; la incorrección se debía fundamentalmente a las falencias de la enseñanza del concepto de función presentes en dichas notas de clase.

Con el fin de entender el problema de la formulación incorrecta, buscamos en la bibliografía especializada de educación matemática, investigaciones sobre los errores comunes en la enseñanza del concepto de función; no obstante, no hallamos ninguna significativa. Por otro lado, dimos con numerosos artículos sobre las dificultades en la enseñanza-aprendizaje del concepto de función (como muestra, véase Oehrtman, M., Carlson, M. y Thompson, P. (2008)) y sobre las consecuencias de definir de maneras distintas conceptos relacionados a las funciones; por ejemplo, la continuidad (véase Jayakody, G. y Zazkis, R. (2015)). Nuestra hipótesis de la falta de investigaciones sobre el problema encontrado en las mencionadas notas es que, en general, este no es común en la enseñanza de las funciones a nivel mundial, parece ser un problema local.
La búsqueda del origen del problema mal planteado nos llevó a formularnos, más bien, una pregunta sobre teoría de funciones. La respuesta llegó en el marco de la Teoría de Conjuntos y del Axioma de Elección, y ese es el tema que tratamos en este artículo.

El ejemplo de las notas de clase mencionadas causante de este artículo es el siguiente:

Redefinir la siguiente función para que sea inyectiva:

$$
f(x)= \begin{cases}x-1 & \text { si } x<1 \\ x^{2}-3 x+2 & \text { si } x \geq 1\end{cases}
$$

En el planteamiento de este problema, encontramos una imprecisión importante: ¿qué significa "redefinir" una función? En ninguna parte de las notas de clase, se encuentra una definición formal ni una explicación intuitiva de "redefinir una función". Ahora bien, en general, en la teoría de funciones, dada una función, la restricción o extensión de esta podría ser entendida como una forma de "redefinición" de la misma. Tanto en el proceso de restringir como de extender una función, el resultado obtenido es una nueva función, diferente de la original aunque "muy parecida a ella", en el sentido de que ambas funciones "comparten" sus imágenes en la intersección de sus dominios; además, el objetivo de la restricción 
o la extensión suele ser el de preservar una o varias propiedades de interés de la función original; por ejemplo, la condición de ser: inyectiva, biyectiva, lineal, continua, continua uniformemente, derivable, integrable o medible, etcétera.

Si nos limitamos, entonces, a entender la "redefinición de una función" como una extensión o una restricción de la misma, solicitar que se "redefina" la función $f$ descrita en el texto universitario, significaría solicitar que busquemos, o bien una extensión o bien una restricción de $f$ que sea inyectiva. Es claro que si $f$ no es inyectiva, una extensión tampoco lo sería. Luego, la solución buscada debería ser una restricción de $f$ que sea inyectiva.

Ahora bien, asuimida la "redefinición de una función" como una restricción de la función original, en el problema encontrado en texto mencionado, nos encontramos con otra dificultad: la función $f$ no está definida de manera correcta, pues no se indica los conjuntos de llegada y salida. En efecto, al no hacerlo, no se está describiendo una función $f$, sino la ley de asignación de una clase infinita de funciones del tipo

$$
\begin{aligned}
\varphi: A & \longrightarrow \mathbb{R} \\
x & \longmapsto \begin{cases}x-1 & \text { si } x<1, \\
x^{2}-3 x+2 & \text { si } x \geq 1,\end{cases}
\end{aligned}
$$

donde $A$ es un subconjunto distinto del vacío de $\mathbb{R}$. Por ejemplo, las funciones cuyas gráficas se muestran en la Figura 1 son tres elementos de esta clase. En este caso, $A$ es $\mathbb{R}$ para $f_{0},(-\infty, 1)$ para $f_{1},[2,+\infty)$ para $f_{2}$ y $(1,+\infty)$ para $f_{3}$. Por tanto, en principio, el problema está mal formulado.
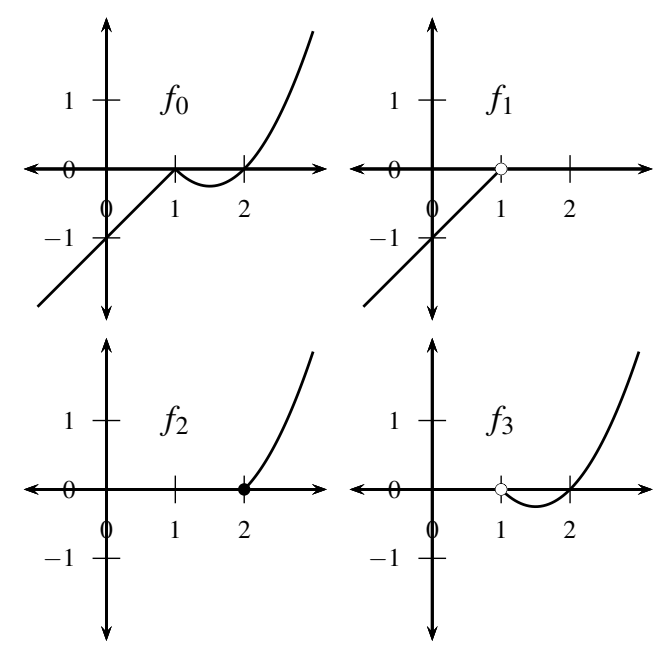

Figura 1. Gráfica de los ejemplos de soluciones al problema.

No obstante, para dar una respuesta a la pregunta del texto, deberíamos elegir un conjunto $A$ particular de modo que la función resultante, a la que llamaremos $f$, no sea inyectiva. Hecho esto, la respuesta al problema es obvia y simple: si tomamos $B=\left\{x_{0}\right\}$, donde $x_{0} \in A$, entonces la restricción de $f$ a $B$ es una función inyectiva, lo que hace que este problema no sea interesante en principio.

Sin embargo, podemos modificar el pedido original para pasar de un problema trivial a uno que implique un instrumento de aprendizaje para los estudiantes. En efecto, una modificación posible es la siguiente: determinar todas las restricciones de $f$ que sean inyectivas. Otra posibilidad es: encontrar una restricción de $f$ de modo que la función original y la restricción tengan la misma imagen.

En este artículo, vamos a ofrecer un análisis de la solución de la segunda posibilidad. Como veremos, una formulación más amplia de la cuestión nos mostrará que esta segunda posibilidad es equivalente al Axioma de Elección, lo que, a su vez, nos indicará que es imposible dar con una solución explícita al nuevo problema. Antes de empezar, presentamos algunas de las definiciones sobre funciones aceptadas por la comunidad matemática actual (Pinter, C., 1971; Mendelson, E., 1997) que serán necesarias para la tarea planteada.

Definición 1 (Función de un conjunto en otro). Si A y B son dos conjuntos no vacíos, $f$ es función de A en B si y solo si

1. $f$ es un subconjunto del producto cartesiano de $A$ y $B$; es decir, si

$$
f \subseteq A \times B
$$

2. Para todo $x \in A$, existe $y \in B$ tal que $(x, y) \in f$.

3. Si $(x, y) \in f y(x, z) \in f$, entonces $y=z$.

Para indicar que " $f$ es una función de A de B", escribiremos

$$
f: A \rightarrow B
$$

Al conjunto A se le conoce como el conjunto de salida de $f$, simplemente, el dominio de $f$, y al conjunto B como el conjunto de llegada de la función.

Si $x \in A$ (es decir, si x está en el dominio de $f$ ), el único elemento $y$ de $B$ tal que $(x, y) \in f$ es representado por $f(x)$ y denominado la imagen de $x$ respecto de $f$. El signo $f(x)$ se lee "efe de $x$ ".

Esta definición precisa (formal) de función de un conjunto en otro puede parafrasearse de la siguiente manera, como se lo hace en muchos textos que, si bien utilizan mecanismos menos formales con fines didácticos, no incurren en imprecisiones matemáticas (por ejemplo, el texto de Stewart, J., Redlin, L. y Watson, S. (2012)):

Si A y B son conjuntos no vacíos, $f$ es una función de $A$ en $B$ si y solo si $f$ es una relación de $A$ en $B(f \subseteq A \times B)$ tal que para todo elemento $x$ de $A$, existe un elemento $y \in B$ que le "corresponde" a $x((x, y) \in f)$ y solo uno (si $(x, y) \in f y(x, z) \in f$, entonces $y=z)$.

Definición 2 (Imagen o recorrido). Sean A y B dos conjuntos no vacíos y $f: A \rightarrow B$. Se define la imagen o recorrido de la función $f$ de $A$ en $B$ al conjunto

$$
\operatorname{img}(f)=\{f(x): x \in A\} .
$$

Definición 3 (Restricción). Sean A y $B$ dos conjuntos no vacíos y $f: A \rightarrow B$. Dado $C \subseteq A$, un conjunto no vacío, la restricción de $f$ a $C$, denotada por $\left.f\right|_{C}$, es la función

$$
\begin{aligned}
\left.f\right|_{C}: C & \longrightarrow B \\
x & \left.\longmapsto f\right|_{C}(x)=f(x) .
\end{aligned}
$$


Definición 4 (Función inyectiva). Sean $A$ y $B$ dos conjuntos no vacíos y $f: A \rightarrow B$. La función $f$ es inyectiva si y solo si para todo $x \in A$ y todo $y \in A$ tales que $x \neq y$, se tiene que $f(x) \neq f(y)$; es decir, si $f(x)=f(y)$, necesariamente $x=y$.

Definición 5 (Función sobreyectiva). Sean A y $B$ dos conjuntos no vacíos y $f: A \rightarrow B$. La función $f$ de $A$ en $B$ es sobreyectiva si y solo si para todo $y \in B$, existe $x \in A$ tal que $y=f(x)$.

Definición 6 (Función biyectiva). Una función es biyectiva si y solo si es inyectiva y sobreyectiva.

\section{Formalización del PROBlema}

En esta sección vamos a formalizar los problemas presentados en la introducción. En primer lugar, no hablaremos de "redefinir una función $f$ para que sea inyectiva", sino que "buscaremos un subconjunto $B$ del dominio de $f$ de manera que la restricción de $f$ a $B$ sea inyectiva". En otras palabras, el problema es el siguiente:

Problema 1. Sean $A \subseteq \mathbb{R}$, diferente del vacío, y $f: A \rightarrow \mathbb{R}$. Se busca $B \subseteq A$, distinto del vacío, tal que la función $\left.f\right|_{B}$ sea inyectiva.

Como ya se dijo, este problema puede tener infinitas soluciones. En primer lugar, si $f$ fuera inyectiva, entonces $\mathscr{P}(A)$ sería el conjunto de todas las soluciones.

Por otra parte, se observa que si $B$ es una solución, cualquier subconjunto de $B$ también lo es. Así, en general, la solución puede no ser única.

Como se vio en la introducción, una solución se obtiene inmediatamente si $B=\left\{x_{0}\right\}$, donde $x_{0} \in A$, pues $\left.f\right|_{B}$ es inyectiva. Esta nos ofrece información importante sobre el problema: existen al menos tantas soluciones como elementos tenga el conjunto $A$.

En la siguiente sección, se darán condiciones para que la solución sea única, se analizarán algunos tipos de soluciones y, con la ayuda del Axioma de Elección, ofreceremos una variedad de soluciones.

Por otro lado, cualquier solución da lugar a una restricción de la función original cuyo recorrido es, en general, diferente del recorrido de la función original. En este sentido, la función obtenida puede ser "bastante diferente" de la función dada inicialmente. Con el fin de obtener una solución que dé lugar a una función lo más "parecida" a la original, el siguiente problema es el resultado de incluir la condición de que la función conserve el recorrido de la función original:

Problema 2. Sean $A \subseteq \mathbb{R}$, diferente del vacío, y $f: A \rightarrow \mathbb{R}$. Se busca $B \subseteq A$, distinto del vacío, tal que la función $\left.f\right|_{B}$ sea inyectiva $y \operatorname{img}\left(\left.f\right|_{B}\right)=\operatorname{img}(f)$.

En la última sección de este artículo, se demostrará que este enunciado es equivalente al Axioma de Elección (véase la página 55), cuando la familia $\left\{A_{i}\right\}_{i \in I}$ es de conjuntos disjuntos de números reales.

A continuación, presentamos las definiciones necesarias para el estudio de los dos problemas (ver Pinter, C. (1971) y Mendelson, E. (1997)).
Definición 7 (Conjunto transitivo). Un conjunto A es transitivo si y solo si $x \in A$ implica $x \subseteq A$; es decir, todo elemento de A es también un subconjunto de $A$.

Definición 8 (Conjunto bien ordenado). Si $(A, \leq)$ es un conjunto con un orden parcial, se dice que está bien ordenado si y solo si todo subconjunto de A distinto del vacío tiene elemento mínimo; es decir, si existe $m \in A$ tal que para todo $x \in A$, se tiene que $m \leq x$.

Definición 9 (Número ordinal). Un conjunto $\alpha$ es un número ordinal si y solo si $\alpha$ es un conjunto transitivo $y\left(\alpha, \leq_{\epsilon}\right)$ está bien ordenado, donde $x \leq_{\in}$ y si y solo si

$$
x \in y \quad \vee \quad x=y,
$$

para todo $x$ y todo y elementos de $\alpha$.

Definición 10 (Igual, menor o igual y menor cardinalidad). Dos conjunto $A$ y $B$ tienen igual cardinalidad si y solo si existe una función biyectiva $f: A \rightarrow B$; en ese caso, se escribirá $|A|=|B|$. Si existe una función inyectiva $g: A \rightarrow B$ se dirá que la cardinalidad de A es menor o igual que la de $B$ y se escribirá $|A| \leq|B|$. Finalmente, se dirá que que la cardinalidad de A es menor que la de $B$ y se notará $|A|<|B|$, si $|A| \leq|B|$ pero $|A| \neq|B|$.

Definición 11 (Número cardinal). Si $\alpha$ es un número ordinal, se dirá que es un número cardinal si y solo si para todo $\beta \in \alpha$, se tiene que $|\beta|<|\alpha|$. Además, dado un conjunto $A$ y un número cardinal $\alpha$, si $|A|=|\alpha|$, se denotará simplemente $|A|=\alpha$ y se dirá que la cardinalidad de A es $\alpha$.

Utilizaremos las siguientes notaciones: si $E$ es un conjunto y $\alpha$ un número cardinal, notaremos con $E^{(\alpha)}$ el conjunto de todos los subconjuntos de $E$ cuya cardinalidad es $\alpha$ :

$$
E^{(\alpha)}=\{B \subseteq E:|B|=\alpha\}
$$

Con $\aleph_{0}$ indicaremos la cardinalidad del conjunto de los números naturales $\mathbb{N}$ y con $c$ la del conjunto de los números reales $\mathbb{R}$.

Finalmente, el siguiente enunciado es la versión del Axioma de Elección que utilizaremos en este artículo:

Axioma 1 (Axioma de Elección). Sean A un conjunto y $\left\{A_{i}\right\}_{i \in I}$ una familia de subconjuntos no vacíos de A, donde I también es un conjunto. Se tiene que existe una función $g: I \rightarrow$ A tal que

$$
g(i) \in A_{i}
$$

para todo $i \in I$.

\section{Análisis del Problema 1}

En esta sección, analizamos las soluciones del Problema 1. En primer lugar, como se mencionó anteriormente, los elementos del conjunto

$$
A^{(1)}=\{\{a\} \subseteq A: a \in A\}
$$

son soluciones triviales del problema. Por tanto, existen al menos tantas soluciones como elementos tenga $A$. Luego, si $A$ tiene más 
de un elemento, la solución del Problema 1 no es única. Recíprocamente, si $A$ es un conjunto unitario, tenemos que

$$
A^{(1)}=\{A\}
$$

con lo cual obtenemos el siguiente resultado:

Proposición 1. El Problema 1 tiene una única solución si y solo si la cardinalidad de A es 1.

Si $A$ tiene más de un elemento, tenemos más de una solución: todos los elementos de $A^{(1)}$. No obstante, todas estas soluciones pueden ser vistas como una sola solución: la clase de todas las soluciones que tienen cardinalidad 1. En particular, si $f$ es constante, este tipo de solución es la única que existe para el Problema 1.

Proposición 2. El conjunto de soluciones del Problema 1 es $A^{(1)}$ si y solo si f es una función constante.

Demostración. Supongamos que $A^{(1)}$ es el conjunto de soluciones del Problema 1 y que $f$ no es una función constante; por tanto, existen $a, b \in A$ tales que $a \neq b$ y $f(a) \neq f(b)$. Así, tomando $B=$ $\{a, b\}$, tenemos que $\left.f\right|_{B}$ es inyectiva; luego, $B$ es una solución del Problema 1 que no pertenece a $A^{(1)}$, lo cual es contradictorio.

Recíprocamente, supongamos que $f$ es una función constante y que existe $B \subseteq A$ tal que $B \notin A^{(1)}$ y $\left.f\right|_{B}$ es inyectiva. Entonces $B$ tiene más de un elemento. Sean $a, b \in B$ tales que $a \neq b$; dado que $\left.f\right|_{B}$ es inyectiva, tenemos que $f(a) \neq f(b)$, de donde $f$ no es constante, lo cual es imposible.

De esta proposición se deduce fácilmente que el conjunto de soluciones del Problema 1 es $A^{(1)}$ si y solo si $|\operatorname{img}(f)|=1$. No obstante, las soluciones generan funciones que son clases unitarias (tienen únicamente un par ordenado como elemento) que, en general, no son de mayor interés.

Por ello, en lugar de buscar condiciones sobre la imagen de la función $f$ para la existencia de una solución del problema 1 , indaguemos la posibilidad de que existan soluciones que tenga más de un elemento; por ejemplo, saber si existe una solución (y si es única) que tenga una cardinalidad dada. Así, consideremos el siguiente problema.

Problema 3. Sean $A \subseteq \mathbb{R}$, diferente del vacío, $\alpha$ un cardinal $y$ $f: A \rightarrow \mathbb{R}$. Se busca $B \subseteq A$, distinto del vacío, tal que $\left.f\right|_{B}$ sea inyectiva $y|B|=\alpha$.

Las siguientes proposiciones dan las condiciones de existencia y unidad para la solución de este problema.

Proposición 3. Para todo cardinal $\alpha$ tal que $\alpha \leq|\operatorname{img}(f)|$, existe una solución del Problema 3.

Demostración. Sea $\alpha$ un cardinal tal que $\alpha \leq|\operatorname{img}(f)|$, tenemos que existe $C \subseteq \operatorname{img}(f)$ tal que $|C|=\alpha$. Tomemos la familia

$$
\left\{f^{-1}(\{y\})\right\}_{y \in C}
$$

la cual está formada por subconjuntos no vacíos de $A$, pues $C \subseteq$ $\operatorname{img}(f)$. Por el Axioma de Elección, existe una función $g: C \rightarrow A$ tal que $g(y) \in f^{-1}(\{y\})$ para todo $y \in C$. Ahora, definamos $B=$ $\operatorname{img}(g) \subseteq A$. Luego, $\left.f\right|_{B}$ es inyectiva, pues si $a, b \in B$ son tales que $f(a)=f(b)$, tenemos que existen $u, v \in C$ tales que

$$
a=g(u) \quad \text { y } \quad b=g(v),
$$

de donde, $a \in f^{-1}(\{u\})$ y $b \in f^{-1}(\{v\})$; es decir,

$$
f(a)=u \quad \text { y } \quad f(b)=v,
$$

luego, $u=v$ y, por tanto, $a=g(u)=g(v)=b$. Así, $B$ es una solución al Problema 1.

Por otro lado, tenemos que $\operatorname{img}\left(\left.f\right|_{B}\right)=C$. En efecto, si $u \in C$, tenemos que $g(u) \in B$, de donde $f(g(u)) \in \operatorname{img}\left(\left.f\right|_{B}\right)$, pero, como $g(u) \in f^{-1}(\{u\}), f(g(u))=u$; así, $u \in \operatorname{img}\left(\left.f\right|_{B}\right)$. Recíprocamente, si $u \in \operatorname{img}\left(\left.f\right|_{B}\right)$, existe $v \in B$ tal que $f(v)=u$ y, como $v \in B=\operatorname{img}(g)$, existe $w \in C$ tal que $g(w)=v$; por tanto, $f\left((g(w))=u\right.$; pero, $g(w) \in f^{-1}(\{w\})$, de donde $f(g(w))=w$; así, $u=w \mathrm{y}$, con ello, concluimos que $u \in C$.

En resumen, $\left.f\right|_{B}$ es inyectiva y, por tanto, $|B|=\left|\operatorname{img}\left(\left.f\right|_{B}\right)\right|=$ $|C|=\alpha$; así, $B$ es una solución del Problema 3 .

La Proposición 3 asegura la existencia de una solución al Problema 3 siempre y cuando $\alpha \leq|\operatorname{img}(f)|$. Por otra parte, si $\alpha>$ $|\operatorname{img}(f)|$, se ve inmediatamente que el Problema 3 no tiene solución. Si $\alpha$ es finito, en la demostración de la Proposición 3, no es necesario el uso del Axioma de Elección y se puede obtener una solución explícita. En el caso de que $\alpha$ sea infinito, en general, el Problema 3 podría no tener una solución explícita (este sería el caso si el Axioma de Elección fuera esencial en la demostración de la existencia de una solución).

A continuación, presentamos algunas características para que el Problema 3 tenga solución única.

Proposición 4. Sea $\alpha \leq|\operatorname{img}(f)|$ un cardinal finito. Si el Problema 3 tiene solución única, esa solución es A y, por tanto, la función $f$ es inyectiva.

Demostración. Supongamos que el Problema 3 tiene solución única y sea $B$ su solución; es decir, $\left.f\right|_{B}$ es inyectiva. Supongamos también que existe $u \in A$ tal que $u \notin B$; se tendrían dos posibilidades para $f(u)$ :

1. Si $f(u) \in f(B)$, existe $v \in B$ tal que $f(v)=f(u)$; luego, tomando $C=(B \backslash\{v\}) \cup\{u\}$, tenemos que $|B|=|C|$ y que $\left.f\right|_{C}$ es inyectiva; por tanto, $C$ sería una solución del problema, lo cual es imposible pues $C \neq B$.

2. Si $f(u) \notin f(B)$, tomemos cualquier elemento $v$ en $B$ y $C$ igual que en el caso anterior; entonces $|B|=|C|$ y $\left.f\right|_{C}$ es inyectiva. Así $C$ sería solución del problema, lo cual es imposible pues $C \neq B$.

Luego, $B=A$; es decir, $A$ es una solución del problema y, por tanto, $f$ es inyectiva, pues $\left.f\right|_{A}=f$.

Es inmediato ver que, si $\alpha=|A|$ es finito, la única posibilidad para que Problema 3 tenga solución es que $f$ sea inyectiva, y su única 
solución sería $A$. A continuación veamos lo que sucede cuando $\alpha$ es infinito.

Proposición 5. El Problema 3 tiene infinitas soluciones si $\alpha \leq$ $|\operatorname{img}(f)| y \alpha$ es un cardinal infinito.

Demostración. Por la proposición3, el Problema 3 tiene al menos una solución; sea $B \subseteq A$ una de ellas. Dado que $|B|=\alpha$ es infinito, para cualquier $u \in B$, el conjunto $C_{u}=B \backslash\{u\}$ tiene cardinalidad $\alpha$, es diferente de $B$ y también es una solución del problema. Así, cada elemento de la familia infinita

$$
\left\{C_{u}\right\}_{u \in B}
$$

es una solución del Problema 3.

\section{Análisis del Problema 2}

En esta sección, analizamos las soluciones del Problema 2. La siguiente proposición indica el caso en el cual el problema tiene solución única.

Proposición 6. El Problema 2 tiene solución única si y solo si $f$ es inyectiva; en este caso, la solución es el conjunto A.

Demostración. Supongamos que $B$ es la única solución del Problema 2 y que existe $u \in A$ tal que $u \notin B$; dado que $f(u) \in$ $\operatorname{img}(f)=\operatorname{img}\left(\left.f\right|_{B}\right)$, existe $v \in B$ tal que $f(u)=f(v)$. Tomemos $C=(B \backslash\{v\}) \cup\{u\}$, tenemos que $C$ es una solución del problema, lo cual es imposible pues $B \neq C$; por tanto, $B=A$, de donde $f=\left.f\right|_{A}$ es inyectiva.

Recíprocamente, si $f$ es inyectiva, $A$ es una solución. Supongamos que $B$ también es una solución y que existe $u \in A$ tal que $u \notin B$. Dado que $f$ es inyectiva, tenemos que $f(u) \notin \operatorname{img}\left(\left.f\right|_{B}\right)$, por lo tanto img $\left(\left.f\right|_{B}\right) \neq \operatorname{img}(f)$, lo cual es imposible; por tanto $B=A$; así $A$ es la única solución del Problema 2.

Para analizar la existencia de una solución del Problema 2, requerimos la siguiente proposición, que es una consecuencia del Axioma de Elección.

Axioma 2 (Axioma de Elección en $\mathbb{R}$ ). Sea $\left\{A_{i}\right\}_{i \in I}$ una familia de subconjuntos no vacíos de $\mathbb{R}$, disjuntos dos a dos, donde $I \subseteq \mathbb{R}$. Existe una función $g: I \rightarrow \mathbb{R}$ tal que $g(i) \in A_{i}$ para todo $i \in I$.

Esta proposición no es equivalente al Axioma de Elección, pero probaremos que es equivalente al Problema 2.

Proposición 7. El Problema 2 siempre tiene solución.

Demostración. Tomemos la familia

$$
\left\{f^{-1}(\{y\})\right\}_{y \in \operatorname{img}(f)},
$$

la cual está formada por subconjuntos no vacíos de $A$. Por el Axioma de Elección en $\mathbb{R}$, existe una función $g: \operatorname{img}(f) \rightarrow A$ tal que $g(y) \in f^{-1}(\{y\})$ para todo $y \in \operatorname{img}(f)$.
El conjunto $B=\operatorname{img}(g) \subseteq A$ es solución. En efecto, $\left.f\right|_{B}$ es inyectiva, ya que si $a, b \in B$ tales que $f(a)=f(b)$; entonces existen $u, v \in C$ tales que

$$
a=g(u) \quad \text { y } \quad b=g(v),
$$

por lo tanto, $a \in f^{-1}(\{u\})$ y $b \in f^{-1}(\{v\})$; es decir,

$$
f(a)=u \quad \text { y } \quad f(b)=v,
$$

de donde, $u=v$ y, por tanto, $a=g(u)=g(v)=b$.

También tenemos que $\operatorname{img}\left(\left.f\right|_{B}\right)=\operatorname{img}(f)$, pues es inmediato que $\operatorname{img}\left(\left.f\right|_{B}\right) \subseteq \operatorname{img}(f)$, y si $u \in \operatorname{img}(f), g(u) \in B$; por tanto, $f(g(u)) \in \operatorname{img}\left(\left.f\right|_{B}\right)$. Finalmente, como $g(u) \in f^{-1}(\{u\})$, tenemos que $f(g(u))=u \mathrm{y}$, por tanto, $u \in \operatorname{img}\left(\left.f\right|_{B}\right)$. Con esto, se tiene que $B$ es una solución del problema.

Proposición 8. La existencia de solución para el Problema 2 implica el Axioma de Elección en $\mathbb{R}$.

Demostración. Sea $\left\{A_{i}\right\}_{i \in I}$ una familia de subconjuntos no vacíos de $\mathbb{R}$, disjuntos dos a dos, donde $I \subseteq \mathbb{R}$. Se define la función

$$
f: \bigcup_{i \in I} A_{i} \rightarrow I
$$

tal que $f(x)=i$ si y solo si $x \in A_{i}$. Dado que los conjuntos son disjuntos, la función se encuentra bien definida; además, dado que la familia es de conjuntos no vacíos, se tiene que $\operatorname{img}(f)=I$. Sea $B$ la solución del Problema 2 para esta función. Tenemos que $\operatorname{img}\left(\left.f\right|_{B}\right)=I \mathrm{y}$, por tanto,

$$
\left.f\right|_{B}: B \rightarrow I
$$

es sobreyectiva; luego, si $g=\left.f\right|_{B} ^{-1}$, obtenemos que

$$
g: I \rightarrow B,
$$

es una función tal que $g(i) \in A_{i}$ para todo $i \in I$.

\section{CONCLUSIONES}

La falta de precisión con que se definen y utilizan los conceptos matemáticos en los cursos de Cálculo en los primeros años de universidad, da lugar al planteamiento de problemas falsos (como el mostrado en este artículo) que, una vez formulados adecuadamente, o bien se convierten en problemas triviales, o bien tienen soluciones que no podrán ser abordadas en este nivel de estudios por los estudiantes.

El problema obtenido (a partir del problema falso) que hemos analizado en este artículo nos muestra la necesidad de que los cursos de formación básica en Matemáticas para las ingenierías deberían manejar de manera consistente los conceptos básicos como los de conjunto, función y número real, entre otros, aunque no los presenten necesariamente con toda la formalidad. 


\section{REFERENCIAS}

Cohen, P. (1963). The Independence of the Continuum Hypothesis. Proc. Natl. Acad. Sci. 50(6). 1143-1148. https://doi.org/10.1073/pnas.50.6.1143

Herrlich, H. (2006). Axiom of Choice. Países Bajos: SpringerVerlag.

Jayakody, G. y Zazkis, R. (2015). Continuos problem of function continuity. For the Learning of Mathematics 35(1), 8-14.

Jech, T. (1973). The Axiom of Choice. Estados Unidos de América: Dover Publications.

Mendelson, C. (1997). Introduction to Mathematical Logic. Estados Unidos de América: Springer-Verlag.

Oehrtman, M., Carlson, M. y Thompson, P. (2008). Foundational Reasoning Abilities that Promote Coherence in Students' Function Understanding. En M. Carlson, y C. Reasmussen (Eds.), Making the Connection Research and Teaching in Undergraduate Mathematics Education (pp. 27-41). Estados Unidos de América: The Mathematical Association of America.

Pinter, C. (1971). Set Theory. Estados Unidos de América: Addison-Wesley.

Stewart, J., Redlin, L. y Watson, S. (2012). Precálculo. México: Cengage Learning.

\section{BIOGRAFÍAS}

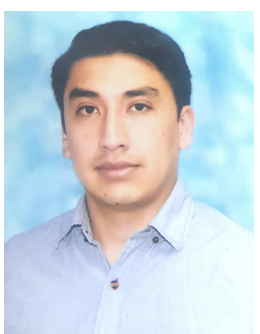

Andrés Esteban Merino Toapanta. Profesor titular a tiempo completo de la Escuela de Ciencias Físicas y Matemática de la Pontificia Universidad Católica del Ecuador, graduado de Matemático en la Escuela Politécnica Nacional y de Magíster en Matemáticas Puras y Aplicadas en la Universidad Central del Ecuador. Sus campos de investigación son la Teoría Descriptiva de Conjunto, Fundamentos de la Matemática y Educación Matemática. https://orcid.org/0000-0002-5404-918X

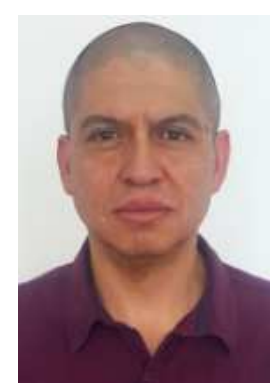

Juan Carlos Trujillo Ortega. Profesor principal a tiempo completo del departamento de Matemática de la Facultad de Ciencias de la Escuela Politécnica Nacional (EPN). Graduado en Matemáticas puras en la EPN (1990) y de Magíster en Informática por la EPN-UASB (1998). Desde 2012, es coordinador del programa CLAVEMAT de la EPN. Sus campos de investigación son los Fundamentos de la Matemática y la Educación Matemática.

https://orcid.org/0000-0002-9017-3137 